\title{
Simulation analysis based on the dual spectrum characteristic algorithm of communication radio station
}

\author{
Wenle Yuan, Xuanmin Lu, Wensheng Luo, Jinjie Cao
}

School of Electronics and Information, Northwestern Polytechnical University, Xi'an, 710072, China.

\begin{abstract}
In order to study the characteristics of double spectrum radio signals, based on higher order statistics and the double spectrum definition and properties, using two kinds of direct and indirect spectrum feature extraction algorithm, and two kinds of radio signal spectrum estimation is simulated. Therefore, it is concluded that the higher order statistics to inhibit Gauss process.
\end{abstract}

Keywords: Double spectrum; Higher-order Statistics; Fourier; feature extraction; pattern identification.

\section{Introduction}

Fingerprint recognition of communication signals includes three processes: preprocessing, feature extraction and classification. The related research abroad focuses on transient signal (i.e. radio signal non stable working condition) boot signal, using the method of analysis of feature extraction to realize radio individual identification, and in-depth research on the transient signal form some of the products. They mainly use radiation sources based on "turn-on" transient feature analysis method to classify this method to the transient features of the integrity of the Yan The requirement and similarity of transient characteristics and noise brought the transient characteristics of interception, extraction difficulty, limits the application of this method; another method of pseudo random sequence is embedded in the signal radiation identification of individual identity in the transmitter based on.

\section{Pattern Recognition and Higher order statistics theory}

\subsection{Pattern Recognition}

Pattern recognition is according to the characteristics or attributes of the research object, the use of machine system with computer as the center, using some analysis algorithm finds its category system should enable the classification result as much as possible in line with the facts. The principle of pattern recognition system and recognition process as shown in Figure 1:

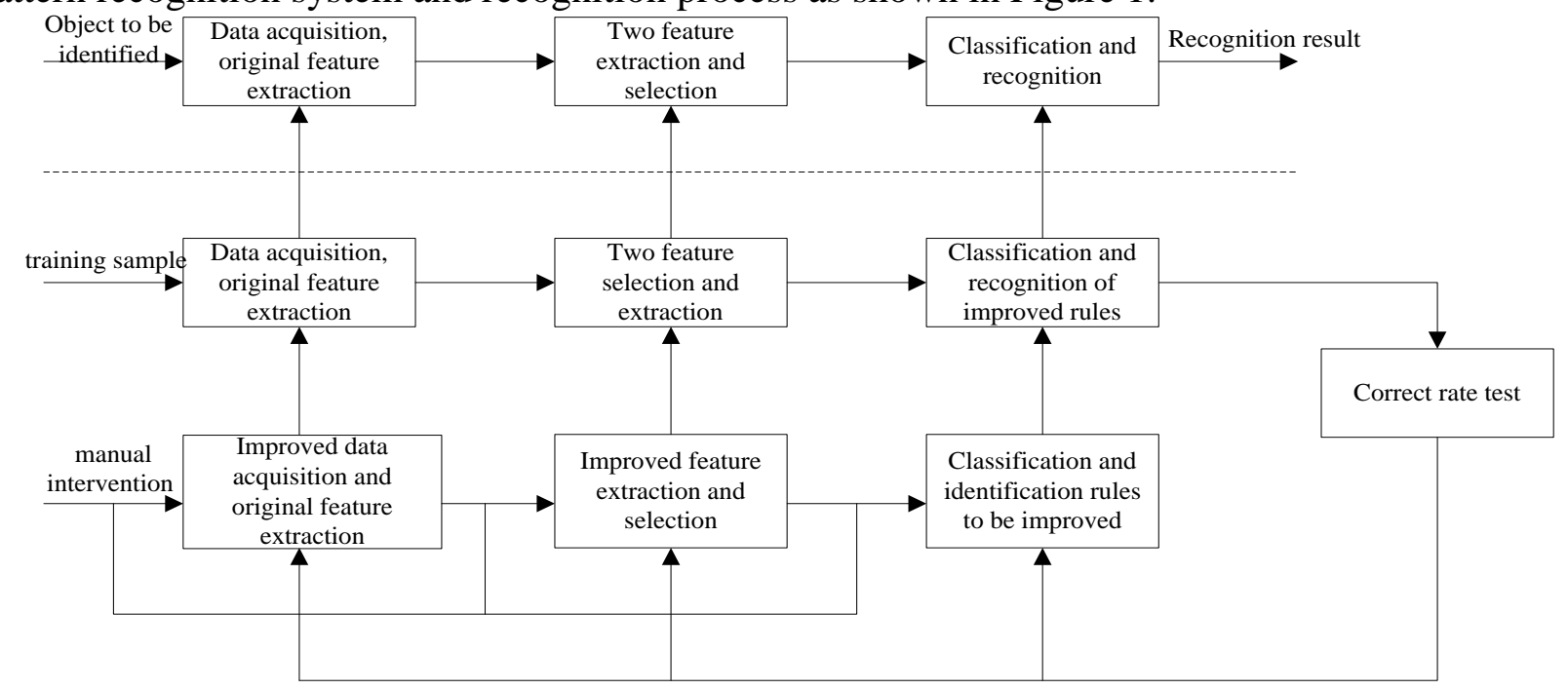

Figure 1 pattern recognition system and recognition process schematic

According to the classification of the object itself has many features, extracted from help classified information is the key to ensure the correct classification. The classification complexity in classification does not exist in the objective standard, any classification of a subjective nature. The 
communication process of radiation source is divided into two stages of design and decision. The design refers to the use of a certain number of samples (training set or learning set) classification, as shown in Figure 2.

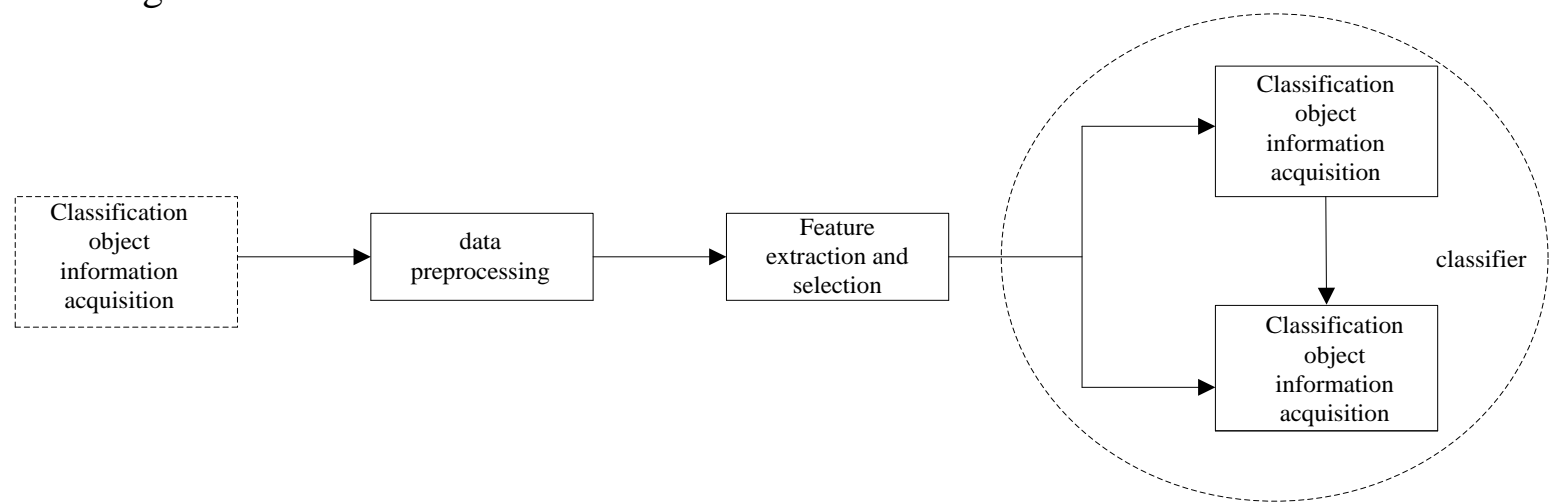

Figure 2 communication radiation source individual identification principle block diagram

Access to information is obtained by measuring, sampling and quantization method can use matrix or vector representation of object data; data preprocessing is to remove the noise, enhance the useful information; according to the specific classification problems with different feature extraction methods, then decided to choose the target classification, classification feature is the key to achieve the classification, properties of this process according to the specific field, combined with prior knowledge and experimental data.

\subsection{Higher order statistics theory}

\subsubsection{Higher order moment and higher order}

Defining the first characteristic function of 3 random variables $\Phi(\omega)$ the order of the origin is equal to the random variable $x$ of $k$ order moment $m_{k}$, at present

$$
m_{k}=\left.\Phi^{k}(\omega)\right|_{\omega=0}=E\left[x^{k}\right]=\int_{-\infty}^{+\infty} x^{k} f(x) d x
$$

Define 4 random variables $x$ second characteristic function $\varphi(\omega)$ at the origin of $k$ the first derivative is equal to the random variable $x$ of $k$ order accumulation $c_{k}$, at present

$$
c_{k}=\left.\varphi(\omega)\right|_{\omega=0}
$$

To generalize the definition of higher order and higher order of the random variables, the definition of higher order moments and higher order of the random vectors can be defined.

Define $5\{x(n)\}$ is zero mean value $k$ order stationary process, the order moment of the process is defined as:

$$
m_{k x}\left(\tau_{1}, \tau_{2}, \ldots, \tau_{k-1}\right)=\operatorname{mom}\left\{x(n), x\left(n+\tau_{1}\right), \ldots, x\left(n+\tau_{k-1}\right)\right\}
$$

But $k$ order is defined as

$$
c_{k x}\left(\tau_{1,} \tau_{2}, \ldots, \tau_{k-1}\right)=\operatorname{cum}\left\{x(n), x\left(n+\tau_{1}\right), \ldots, x\left(n+\tau_{k-1}\right)\right\}
$$

\subsubsection{Higher order moment and higher order accumulation of Gauss process}

Gauss distribution of random variables and random process in probability theory, mathematical statistics and time series has a very important role in [2]. In this section will focus on the analysis of the Gauss stochastic process of high order moment and high order accumulation.

The cumulative generating function is

$$
\phi_{\xi}(\omega)=\ln \varphi_{\xi}(\omega)=-\frac{\sigma^{2} \omega^{2}}{2}
$$

The general expressions of the higher order moments are derived by using the defined formula (8) and (9):

$$
m_{k}=E\left(\xi^{k}\right)=\left\{\begin{array}{c}
\phi \\
1,3, \ldots,(k-1) \sigma^{2}
\end{array}\right.
$$


Here is $n$ higher order moments and higher order of the dimensional random vectors, Set random vector $x=\left[x_{1}, x_{2}, \ldots, x_{n}\right]^{\mathrm{T}}$ Gauss distribution, its mean vector is $\beta=\left[\beta_{1}, \beta_{2}, \ldots, \beta_{n}\right]^{\mathrm{T}}$, covariance matrix

$$
Q=\left[\begin{array}{cccc}
q_{11} & q_{12} & \ldots & q_{1 n} \\
q_{21} & q_{22} & \ldots & q_{2 n} \\
\vdots & \vdots & \vdots & \vdots \\
q_{n 1} & q_{n 2} & \ldots & q_{n n}
\end{array}\right]
$$

Nonnegative positive definite, among $q_{i j}=E\left\{\left(x_{i}-\beta_{i}\right)\left(x_{j}-\beta_{j}\right)\right\}, i, j=1,2,3, \ldots, n$

Random variable $x$ joint probability density

$$
f(x)=\frac{1}{\sqrt[n]{2 \pi} \sqrt{|q|}} e^{-(1 / 2)(x-\beta)^{\mathrm{T}} e^{-1}(x-\beta)}
$$

All of the above shows that the higher order of Gauss's process is equal to zero, and higher order moments do not possess this property, that is, the higher order moment of Gauss process is not identical to zero.

\subsubsection{The properties and algorithms of the double spectrum}

Multi spectral static signal sequence $x(n)$ of $k$ the order of the cumulative quantity is defined as follows:

$$
m_{k x}\left(\tau_{1,} \tau_{2}, \ldots, \tau_{k-1}\right)=E\left\{x(n), x\left(n+\tau_{1}\right), \ldots, x\left(n+\tau_{k-1}\right)\right\}
$$

$k$ the order power spectrum is defined as follows:

$$
M_{k x}\left(\omega_{1}, \omega_{2}, \ldots, \omega_{k-1}\right)=\sum_{\tau_{1}=-\infty}^{+\infty} \ldots \sum_{\tau_{k-1}=-\infty}^{+\infty} m_{k x}\left(\tau_{1}, \tau_{2}, \ldots, \tau_{k-1}\right) \exp \left[-j \sum_{i=1}^{k-1} \omega_{i} \tau_{i}\right]
$$

1) Double spectrum $B_{x}\left(\omega_{1}, \omega_{2}\right)$ Is generally complex, that is, with the amplitude and phase, that is, the

$\mathrm{B}_{x}\left(\omega_{1}, \omega_{2}\right)=\mid \mathrm{B}_{x}\left(\omega_{1}, \omega_{2}\right) \exp \left[j \varphi_{\mathrm{B}}\left(\omega_{1}, \omega_{2}\right)\right]$

2) Double spectrum $\mathrm{B}_{x}\left(\omega_{1}, \omega_{2}\right)$ with the following symmetrical form:

$\mathrm{B}_{x}\left(\omega_{1}, \omega_{2}\right)=\mathrm{B}_{x}\left(\omega_{2}, \omega_{1}\right)=\mathrm{B}_{x}^{*}\left(-\omega_{21},-\omega_{1}\right)=\mathrm{B}_{x}\left(\omega_{2},-\omega_{1}-\omega_{2}\right)$

3) Double spectrum $\mathrm{B}_{x}\left(\omega_{1}, \omega_{2}\right)$ is a periodic function, with a period of $2 \pi$, the

$\mathrm{B}_{x}\left(\omega_{1}, \omega_{2}\right)=\mathrm{B}_{x}\left(\omega_{1}+2 \pi, \omega_{2}+2 \pi\right)$

For discrete time series signals $x(n)$, an infinite parallel symmetric line family is introduced as a result of sampling: $2 \omega_{1}+\omega_{2}=n_{1} \omega_{s}, 2 \omega_{1}-\omega_{2}=n_{1} \omega_{s}, \omega_{1}+2 \omega_{2}=n_{1} \omega_{s}, \omega_{1}-2 \omega_{2}=n \omega_{s}$, among them, $\omega_{s}=2 \pi f_{s}$, for the sampling angle frequency, the main field of the above continuous signal $\mathrm{C}$ first, symmetric lines $2 \omega_{1}+\omega_{2}=\omega_{s}$ the cut, so, discrete time signal $x(n)$ of double spectrum $\hat{\mathrm{B}}_{x}\left(\omega_{1}, \omega_{2}\right)$ the main field is shown in Figure $3 C=\left\{\omega_{1}, \omega_{2}: 0 \leq \omega_{1} \leq \omega_{s} / 2, \omega_{2} \leq \omega_{1}, \omega_{1}+\omega_{2}=\omega_{s}\right\}$. 


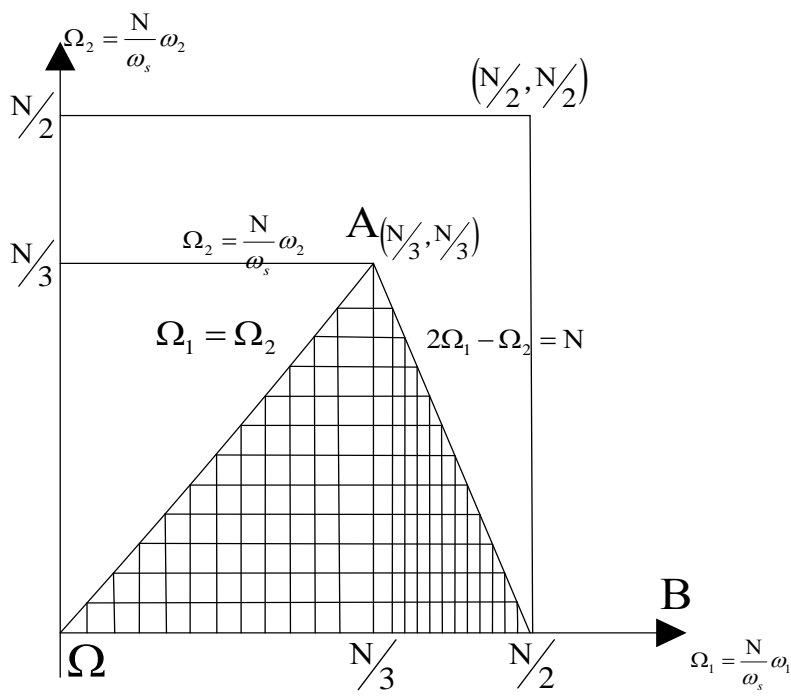

Figure 3 discrete time signal dual spectrum main field

Dual spectrum algorithm:

Direct and indirect methods are commonly used in the two kinds of dual spectrum algorithm, the following is the specific algorithm of the two methods. $x(1), x(2), \ldots, x(n)$ for a set of observational data, and $f_{s}$ Sampling frequency, $\Delta_{0}=f_{s} / \mathrm{N}_{0}$ is the interval between the two frequency sampling points required in the dual spectral region along the horizontal and vertical directions. $\mathrm{N}_{0}$ is the total number of frequency samples.

(1) Direct method

Steps are as follows:

The data segment is given $\mathrm{K}$ segment, each section contains $\mathrm{M}$ observation samples, $\mathrm{N}=\mathrm{KM}$, and the average value of each segment is subtracted from the data. If necessary, zero is added to the data in order to meet the requirements of the general length $m$ of the FFT.

1) Calculated DFT coefficient

$$
\mathrm{Y}^{(1)}(\lambda)=\frac{1}{\mathrm{M}} \sum_{n=0}^{\mathrm{M}-1} y^{(i)}(n) \exp (-2 j 2 \pi n \lambda / \mathrm{M}) \quad \lambda=0,1, \ldots, \mathrm{M} / 2 ; i=1,2, \ldots, \mathrm{K}
$$

Among $\left\{y^{(i)}(n), n=1,2, \ldots, \mathrm{M}-1\right\}$ is the first $i$ data segment.

2) Calculation of the DFT coefficient of the three weight correlation:

$$
\hat{b}\left(\lambda_{1}, \lambda_{2}\right)=\frac{1}{\Delta_{0}^{2}} \sum_{k_{1}=-L_{1}}^{L_{1}} \sum_{k_{2}=-L_{1}}^{L_{1}} \mathrm{Y}^{(i)}\left(\lambda_{1}+k_{1}\right) \mathrm{Y}^{i}\left(\lambda_{2}+k_{2}\right) \times \mathrm{Y}^{(i)}\left(-\lambda_{1}-\lambda_{2}-k_{1}-k_{2}\right)
$$

Among $0 \leq \lambda_{2} \leq \lambda_{1}, \quad \lambda_{1}+\lambda_{2} \leq f_{s} / 2, \mathrm{~N}_{0}, L_{1}$ should meet $\mathrm{M}=\left(2 L_{1}+1\right) \mathrm{N}_{0}$ value.

3) The double spectrum of the given data is given by the average value of the $\mathrm{K}$ segment.

$\hat{\mathrm{B}_{D}}\left(\begin{array}{c}- \\ \omega_{1}, \omega_{2}\end{array}\right)=\frac{1}{\mathrm{~K}} \sum_{i=1}^{\mathrm{K}} \hat{b_{i}}\left(\begin{array}{c}- \\ \omega_{1}, \omega_{2}\end{array}\right)$

Among

$\overline{\omega_{1}}=\left(\frac{2 \pi f_{s}}{\mathrm{~N}_{0}}\right) \lambda_{1}, \overline{\omega_{2}}=\left(\frac{2 \pi f_{s}}{\mathrm{~N}_{0}}\right) \lambda_{2}$

(2) Indirect method

Steps are as follows:

1) Will data $x(1), x(2), \ldots, x(n)$ divided into $\mathrm{k}$ segments, each with $\mathrm{m}$ samples, and subtract the respective mean.

$\left\{x^{(i)}(n), n=1,2, \ldots, \mathrm{M}-1\right\}$ is the first ${ }^{i}$ data segment, an estimated three order cumulant segments: 


$$
c^{(i)}(l, k)=\frac{1}{\mathrm{M}} \sum_{t=\mathrm{M}}^{\mathrm{M}_{2}} x^{(i)}(t) x^{(i)}(t+1) x^{(i)}(t+k), i=1,2, \ldots, k
$$

Type

$$
\begin{aligned}
& \mathrm{M}_{1}=\max (0,-l,-k) \\
& \mathrm{M}_{2}=\min (\mathrm{M}-1, \mathrm{M}-1-l, \mathrm{M}-1-k)
\end{aligned}
$$

2) The average of the three order of all segments is estimated as the three order of the whole observation data set:

$$
\hat{c}(k, 1)=\frac{1}{\mathrm{~K}} \sum_{i=1}^{\mathrm{K}} c^{(i)}(l, k)
$$

3) Generation of double spectrum estimation:

$$
\hat{\mathrm{B}_{\mathrm{IN}}}\left(\overline{\omega_{1}}, \overline{\omega_{2}}\right)=\sum_{l=-L k=-L}^{L} \sum_{c}^{L} \hat{c}(l, k) \bar{\omega}(l, k) \exp \left\{-j\left(\overline{\omega_{1}} l+\overline{\omega_{2}} k\right)\right\}
$$

Type $L<\mathrm{M}-1$, but $\omega(1, k)$ generation of double spectrum estimation:

Due to the symmetry of the (6) use of the three order of the cumulative amount, and in the (8) in the use of the symmetry of the double spectrum, the calculation of indirect algorithm can be greatly decreased.

\section{Analysis of the characteristics of communication signal}

The biggest advantage of higher order statistics can suppress Gauss noise, can provide useful information from signal noise, and effectively improve the recognition rate. The linear assumption, and the traditional Gauss noise signal, stationary or local stationarity, the actual signal noise is a nonlinear, non-Gauss and smooth signal. The high order spectrum can not only restrain the Gauss noise, but also reflect the combined signal phase and phase information. The lotus type with two kinds of signal as an example, the spectrum estimation, such as figure 4:

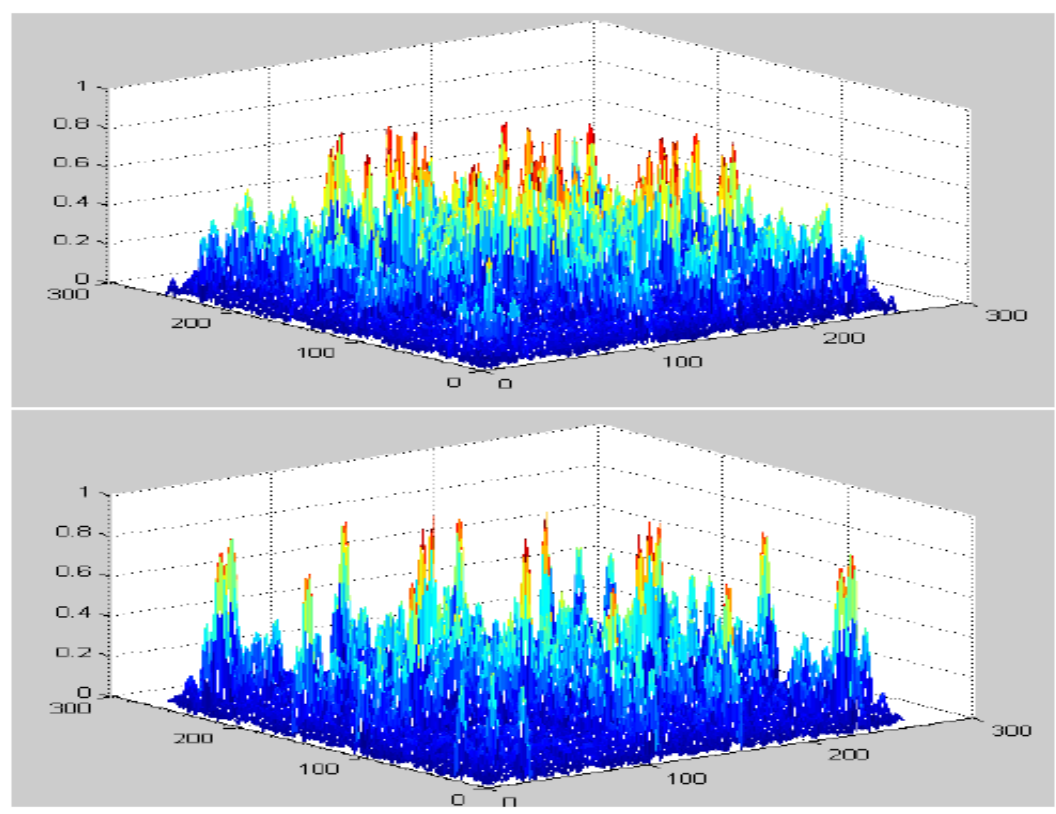

Figure 4 two kinds of radio signals of the dual spectrum

This section of two kinds of radio signals in the double spectrum estimation, noise two signals are non-Gauss components. From the above, 5 different radio signal spectrum can be seen double difference signal noise in the continuous spectrum and intensity and so on. 


\section{Summary}

This paper starts from the characteristic function of random variables, random variables of higher-order moments and cumulates and the corresponding spectrum gives the definition, introduces the Gauss process of higher-order moments and cumulates and some basic properties. Finally, considering the needs of feature extraction, this chapter focuses on the nature of the double spectrum and two kinds of commonly used algorithms, and simulation analysis of double spectrum communication signal noise.

\section{Acknowledgments}

Northwestern Polytechnical University graduate creative new seed fund

\section{References}

[1] J. Toonstra, W. Kinsner. A Radio Transmitter Fingerprinting System ODO-1 [C]. Canadian Conference on Electrical and Computer Engineering, 1996, (1): 60-63.

[2] D. Shaw, W. Kinsner. Multifractal modelling of radio transmitter transients for classification [J]. IEEE Proceedings, WESCANEX 97: Communications, Power and Computing, IEEE, 22-23 May 1997, 306-312.

[3] Xianbin Wang, Yiyan Wu, B. Caron. Transmitter identification using embedded spread spectrum sequences [C]. Proc. International Conference on Communication Technology, ICCT 2003, (2): $1517-1523$.

[4] N. Cristianini and J shawe-Taylao. An Introduction to support vector machines [M]. Cambridge: Cambridge University press, 2000.

[5] V. N. Vpanik and A. Y. Chervoenkis. Theory of Pattern Recognition. Moscow [M]. Russia Nauka, 1974.

[6] John Shawe-Taylor. Kernel Methods for Pattern Analysis [M]. University of Southampton: Cambridge University Press, 2004.

[7] Guicai Zhang, Ruxu Du, Tielin Shi. Extracting Gear Fault Features Using Integrated Bispectrum [J]. Dept of Automation and Computer-Aided Engineering, the Chinese University of Hang Kong, Hong Kong, 2003, 548-553.

[8] Yuerong Wang, Wei Wang, Yuelei Liu, etc. Feature Extracting of Weak Signal in Real-Time Sleeping EEG with Approximate Entropy and Bispectrum Analysis [J]. Institute of Biomedical Engineering, School of Information Science and Engineering, Lanzhou University, 2009:1-4.

[9] Yu Shi, Xian-Da Zhang. Local Bispectra Based High-Resolution Radar Target Classification [J], State Key Laboratory of Intelligent Technology and Systems Dept. of Automation, Tsinghua University, Beijing, 2000:1968-1971. 